\title{
Antimicrobial resistance in Enterococcus spp. isolated from laying hens of backyard poultry flocks
}

\author{
Fabrizio Bertelloni ${ }^{1}$, Claudia Salvadori' ${ }^{1}$ Annalisa Moni ${ }^{2}$, Domenico Cerri', Paolo Mani', \\ Valentina Virginia Ebani ${ }^{1}$ \\ ${ }^{1}$ Department of Veterinary Science, University of Pisa, Italy \\ 2 Dipartimento di Prevenzione, UOC Sanità Animale, Azienda USL Massa Carrara, Italy
}

Bertelloni F, Salvadori C, Moni A, Cerri D, Mani P, Ebani VV. Antimicrobial resistance in Enterococcus spp. isolated from laying hens of backyard poultry flocks. Ann Agric Environ Med. 2015; 22(4): 665-669. doi: 10.5604/12321966.1185771

\begin{abstract}
Introduction and objective. Enterococci belonging to human and animal gastrointestinal flora are widely-distributed in the environment. They are opportunistic bacteria that can cause severe infections, with the ability to acquire, express and transfer antimicrobial resistance. The aim of the present study was to investigate antimicrobial resistance profiles of Enterococcus spp. strains isolated from cloacal swabs of laying hens of small backyard flocks.

Materials and methods. Susceptibility to 21 antimicrobial agents was tested by the disc diffusion method in 115 Enterococcus spp. strains. Vancomycin and ampicillin minimum inhibitory concentrations and high-level aminoglycoside resistance tests were also performed.

Results. Isolates showed resistance mainly to aminoglycosides, eritromycin, fluoroquinoles, tetracycline and nitrofurantoin. $19(16.5 \%)$ isolates showed a high level of resistance to streptomycin, but no high level resistance to gentamycin. No significant resistance was detected for vancomycin. Several strains (45; 39.1\%) showed combined resistance to macrolides, lincosamides and streptogramin B. 61 (53\%) isolates were classified as multidrug-resistant (MDR) and 6 (5.2\%) strains as possibly extensively drug-resistant (XDR). E. faecium was the most prevalent antimicrobial resistant species, followed by $E$. faecalis and E. durans.

Conclusions. The results show that the risk of dissemination of antimicrobial resistant enterococci is related not only to the birds of large commercial flocks, but also to the birds of small backyard flocks. Thus, laying hens of hobby flocks, which share the outside environment with people, could represent a hazard for public health by providing a conduit for the entrance of resistance genes into the community.
\end{abstract}

\section{Key words}

Enterococcus spp., laying hens, antimicrobial resistance, cloacal swabs, hobby poultry

\section{INTRODUCTION}

Enterococci (genus Enterococcus) belong to the gastrointestinal flora of humans and animals and are widely distributed in the environment, such as terrestrial and water habitats [1]. As residents of the human and animal gastrointestinal tract, enterococci bring numerous benefits, such as probiotic activity and bacteriocins production. However, enterococci have raised research interests due to their pathogen role as opportunistic bacteria that can cause severe human infections, mainly as nosocomial infections, and for their strong ability to acquire, express and transfer antimicrobial resistance $[1,2]$. In the gastrointestinal habitat, enterococci are in a suitable position to acquire resistance genes from other commensals, which may further transfer to other more pathogenic bacteria [3]. The clinically most important species in human beings are E. faecalis and E. faecium that can be involved in urinary tract infections, endocarditis, wound infections, sepsis and neonatal infections $[4,5]$. E. faecalis is the most pathogenic species and E. faecium and is the most involved in the acquisition and transfer of antimicrobial resistance [5].

Address for correspondence: Valentina Virginia Ebani, Department of Veterinary Science, University of Pisa, Viale delle Piagge, 2; 56124 Pisa, Italy

E-mail: valentina.virginia.ebani@unipi.it

Received: 10 March 2015; accepted: 14 April 2015
The mechanism for antibiotic resistance can be intrinsic to enterococci or acquired through mutations of intrinsic genes, or horizontal exchange of genetic elements encoding resistance determinants [6]. The latter mechanism is the most important in the antimicrobial resistance of enterococci, and involves mobile genetic elements such as plasmids and transposons [3].

Enterococci are involved also as etiological agents of infections in veterinary medicine, such as mastitis in cattle, diarrhea in swine and cattle, as well as endocarditis, septicemia, spondylitis, and amyloid arthropathy in poultry $[7,8]$.

Humans can be colonized or infected with resistant enterococci through close contact with animals or through consumption of animal products $[9,10]$.

The presence of multidrug resistant enterococci has been detected worldwide on poultry farms in Australia [11], Canada [12,13] and Malaysia [14], as well as in Europe: e.g., Sweden [15], Denmark [16], France [17], the Czech Republic [18] and Lithuania [8]. Continual and careful monitoring programmes are necessary to obtain data on the occurrence and trends in antimicrobial resistance, and consequently, for establishing intervention strategies [19].

The health status of backyard poultry flocks is generally poorly investigated. In particular, studies on the dissemination of multidrug resistant enterococci among commercial poultry have been carried out, but to the best 
of the authors'r knowledge no data are available about this concern in hobby poultry.

The aim of the presented study was to investigate the antimicrobial resistance profiles of Enterococcus spp. strains isolated from cloacal swabs of laying hens of small backyard poultry flocks.

\section{MATERIALS AND METHOD}

Sampling. 157 cloacal swabs were collected from healthy laying hens of 24 different hobby poultry flocks in the Massa Carrara province of central Italy. The birds were geographically separated with no transfer between farms. Backyard flocks were characterized by a small number of raised animals (10-50) that live in open spaces or small fenced areas. Swabs were collected by the random capture of the animals, and the swabs were kept at $4{ }^{\circ} \mathrm{C}$ until bacteriological examinations.

Bacterial isolation. Within 24 hours of collecting, the swabs were sewn directly on Kanamycin Aesculin Azide Agar (KAAA, Oxoid Ltd., Basingstoke, UK) and incubated at $42 \pm 1{ }^{\circ} \mathrm{C}$ for $18-24$ hours. From plates with growth of colonies typical for enterococci, at least one colony was subcultured on KAAA. Isolates were stored at $-80^{\circ} \mathrm{C}$ in Brain Hearth Infusion Broth (BHI, Oxoid) for further investigations.

Antimicrobial susceptibility testing - Disc diffusion method. Isolates were tested by the standard disc diffusion method of Kirby-Bauer [20] on Mueller Hinton Agar (Oxoid) incubated at $35 \pm 1 \mathrm{C}^{\circ}$ for $18-24$ hours. The following antimicrobial molecules (Oxoid) were tested: amoxicillinclavulanic acid $(30 \mu \mathrm{g})$, ampicillin $(10 \mu \mathrm{g})$, cephalothin $(30 \mu \mathrm{g})$, chloramphenicol $(30 \mu \mathrm{g})$, ciprofloxacin $(5 \mu \mathrm{g})$, clindamycin $(2 \mu \mathrm{g})$, enrofloxacin $(5 \mu \mathrm{g})$, eritromycin $(10 \mu \mathrm{g})$, gentamycin $(10 \mu \mathrm{g})$, linezolid $(30 \mu \mathrm{g})$, neomycin $(10 \mu \mathrm{g})$, nitrofurantoin $(300 \mu \mathrm{g})$, oxacillin $(1 \mu \mathrm{g})$, quinupristindalfopristin $(15 \mu \mathrm{g})$, rifampicin $(30 \mu \mathrm{g})$, streptomycin $(10 \mu \mathrm{g})$, teicoplanin $(30 \mu \mathrm{g})$, tetracycline $(30 \mu \mathrm{g})$, trimethoprim $(5 \mu \mathrm{g})$, vancomycin $(30 \mu \mathrm{g})$. Results were interpreted following EUCAST breakpoint Tables and, where not possible, according to NCCLS indications $[21,22]$. References strains E. faecalis ATCC 29212 and E. faecium ATCC 19434 were used as controls.

Minimum inhibitory concentration (MIC). MIC for vancomicin and ampicillin were performed on microplates [23]. Concentrations from $0.5-256 \mu \mathrm{g} / \mathrm{mL}$ were used to test vancomicin MIC, and concentrations $8-256 \mu \mathrm{g} / \mathrm{mL}$ were used for MIC of ampicillin. Microplates were incubated at $37 \pm 1^{\circ} \mathrm{C}$ in a humid chamber.

High level aminoglycoside resistance (HLAR). As indicated by CLSI Performance Standards for antimicrobial susceptibility tests, isolates that showed resistance to gentamicin and/or streptomycin by the disc diffusion method, were tested for resistance to high concentration of gentamicin $(500 \mu \mathrm{g} / \mathrm{mL})$ and streptomycin $(1,000 \mu \mathrm{g} / \mathrm{mL})$ [23].

Classification of acquired resistance. To classify isolated strains for expression of acquired resistance, the standardized international terminology proposed by Magiorakos et al.,
2012 [24] has been used in this study. For enterococci, aminoglycosides, carbapenems, fluoroquinolones, glycopeptides, glycylcyclines, lipopeptides, oxazolidinones, penicillins, streptogramins and tetracycline categories should be tested. Criteria for defining acquired resistance are: multidrug-resistant (MDR) strain when it is non-susceptible to $\geq 1$ agent in $\geq 3$ antimicrobial categories; extensively drugresistant (XDR) strain when it is non-susceptible to $\geq 1$ agent in all but $\leq 2$ categories; and pandrug-resistant (PDR) strain when it is non-susceptible to all antimicrobial agents listed.

Since not all proposed molecules were tested in this study, only MDR or possibly XDR strains could be detected.

Species identification. Enterococcus spp. isolates classified as MDR or possibly XDR was examined for species identification with API 20 STREP (Bio Mérieux Italia, Bagno a Ripoli, Fi, Italy). Apiweb V 1.1.0 software was used as interpretative criteria.

\section{RESULTS}

Bacterial isolation. 115 Enterococcus isolates were obtained from 157 cloacal swabs.

Antimicrobial susceptibility testing - Disc diffusion method. All 115 isolaters were tested for antimicrobial susceptibility with the disc diffusion method; results shown on Table 1. All isolates were not susceptible to oxacillin and most of them were not susceptible to cephalothin (86.9\%) and trimethoprim (95.7\%). Moreover, isolates were more frequently non-susceptible to the aminoglycosides category (100\% of isolates were non-susceptible to neomycin and 93\% were non-susceptible to streptomycin), macrolides ( $87.8 \%$ of isolates were non-susceptible to erythromycin), fluoroquinolones $(86.1 \%$ of isolates were non-susceptible to enrofloxacin) and lincosamides (81.7\% of isolates were nonsusceptible to clindamycin). Over half of the isolates were non-susceptible to ciprofloxacin (70.4\%), tetracycline (65.2\%), gentamicin (60\%) and quinupristin-dalfopristin (53.9\%). A moderate resistance was evident for nitrofurantoin (48.7\%), ampicillin (29.6\%), tigecycline (26.1\%), rifampicin (22.6\%) and chloramphenicol (19.1\%). Only a limited number of isolates were not susceptible to glycopeptides (vancomycin $10 \%$ and teicoplanin 11\%) and to association of amoxicillinclavulanic acid (13\%).

A total of 101 resistance patterns were identified and all Enterococcus spp. isolates were resistant to at least 2 different categories of antibiotics, with 106 (92.17\%) isolates being resistant to 5 or more antibiotics. Two isolates were susceptible only to vancomycin, one isolate was susceptible only to ampicillin and to amoxicillin-clavulanic acid, 6 isolates were susceptible only to 3 tested molecules, and 9 isolates were susceptible to 4 tested antibiotic molecules.

Minimum inhibitory concentration (MIC). All isolates characterized by a non-susceptibility to vancomycin and/or ampicillin with Kirby-Bauer test were tested for determination of MIC of these molecules. For vancomycin, 7 isolates showed $\mathrm{MIC}=1 \mathrm{mg}^{-1}$; one isolate showed $\mathrm{MIC}=2 \mathrm{mg}^{-1}$, and 2 isolates showed $\mathrm{MIC}=4 \mathrm{mg}^{-1}$. For ampicillin, 22 isolates showed $\mathrm{MIC} \leq 8 \mathrm{mg}^{-1}$, 7 isolates with $\mathrm{MIC}=16 \mathrm{mg}^{-1}, 4$ isolates with $\mathrm{MIC}=32 \mathrm{mg}^{-1}$ and one isolate with $\mathrm{MIC}=64 \mathrm{mg}^{-1}$. 
Table 1. Antimicrobial resistance expression of Enterococcus spp. isolates as result by disc diffusion method. Number and percentage of isolates resistant to 21 antibiotics are shown

\begin{tabular}{|c|c|c|c|c|c|c|c|c|}
\hline Antibiotic & $\begin{array}{l}\text { Susceptible } \\
\text { (No. isolates) }\end{array}$ & $\%$ & $\begin{array}{l}\text { Intermediate } \\
\text { (No. isolates) }\end{array}$ & $\%$ & $\begin{array}{c}\text { Resistant } \\
\text { (No. isolates) }\end{array}$ & $\%$ & $\begin{array}{c}\text { No-susceptible: } \\
\text { intermediate + resistant } \\
\text { (No. isolates) }\end{array}$ & $\%$ \\
\hline Amoxicillin-clavulanic acid (AMC) & 100 & 87.0 & 13 & 11.3 & 2 & 1.7 & 15 & 13.0 \\
\hline Ampicillin (AMP) & 81 & 70.4 & 1 & 0.9 & 33 & 28.7 & 34 & 29.6 \\
\hline Cephalotin (KF) & 15 & 13.0 & 20 & 17.4 & 80 & 69.6 & 100 & 86.9 \\
\hline Chloramphenicol (C) & 93 & 80.9 & 13 & 11.3 & 9 & 7.8 & 22 & 19.1 \\
\hline Ciprofloxacin (CIP) & 34 & 29.6 & 61 & 53.0 & 20 & 17.4 & 81 & 70.4 \\
\hline Clindamycin (DA) & 21 & 18.3 & 9 & 7.8 & 85 & 73.9 & 94 & 81.7 \\
\hline Enrofloxacin (ENR) & 16 & 13.9 & 32 & 27.8 & 67 & 58.3 & 99 & 86.1 \\
\hline Eritromycin (E) & 14 & 12.2 & 59 & 51.3 & 42 & 36.5 & 101 & 87.8 \\
\hline Gentamycin (CN) & 46 & 40.0 & 37 & 32.2 & 32 & 27.8 & 69 & 60.0 \\
\hline Linezolid (LZD) & 74 & 64.3 & 15 & 13.0 & 26 & 22.6 & 41 & 35.6 \\
\hline Nitrofurantoin (F) & 59 & 51.3 & 14 & 12.2 & 42 & 36.5 & 56 & 48.7 \\
\hline Oxacillin (OX) & 0 & 0.0 & 0 & 0.0 & 115 & 100 & 115 & 100 \\
\hline Quinupristin-dalfopristin (QD) & 53 & 46.1 & 23 & 20.0 & 39 & 33.9 & 62 & 53.9 \\
\hline Rifampicin (RD) & 89 & 77.4 & 7 & 6.1 & 19 & 16.5 & 26 & 22.6 \\
\hline Streptomycin (S) & 8 & 7.0 & 10 & 8.7 & 97 & 84.3 & 107 & 93.0 \\
\hline Teicoplanin (TEC) & 104 & 90.4 & 9 & 7.8 & 2 & 1.7 & 11 & 9.6 \\
\hline Tetracycline (TE) & 40 & 34.8 & 9 & 7.8 & 66 & 57.4 & 75 & 65.2 \\
\hline Tigecycline (TGC) & 85 & 73.9 & 14 & 12.2 & 16 & 13.9 & 30 & 26.1 \\
\hline Trimethoprim (W) & 5 & 4.3 & 83 & 72.2 & 27 & 23.5 & 110 & 95.7 \\
\hline Vancomycin (VA) & 105 & 91.3 & 7 & 6.1 & 3 & 2.6 & 10 & 8.7 \\
\hline
\end{tabular}

Table 2. Resistance patterns of strains identified as E. faecalis and classified as MDR

\begin{tabular}{lc}
\hline Resistance pattern & Strain \\
\hline CIP; KF; DA; ENR; E; CN; N; QD; RD; S; TE & $37 ; 38$ \\
\hline KF; DA; ENR; E; CN; LZD; N; F; QD; RD; S; TE; TGC & 39 \\
\hline KF; CIP; DA; ENR; E; CN; LZD; N; QD; RD; TE; TGC & 46 \\
\hline KF; C; CIP; DA; ENR; E; CN; LZD N; F; QD; RD; S (HLRA); TE; TGC & 43 \\
\hline CIP; DA; ENR; E; CN; N; QD; S (HLRA); TE & 229 \\
\hline CIP; DA; ENR; E; CN; N; QD; TE; TGC & 40 \\
\hline AMC; AMP; KF; CIP; DA; ENR; CN; N; QD; TGC & 65 \\
\hline AMP; KF; C; CIP; DA; ENR; E; CN; LZD; N; QD; TE; TGC & 92 \\
\hline CIP; DA; ENR; E; CN; LZD; N; QD; TE & 219 \\
\hline CIP; DA; ENR; E; CN; LZD; N; QD; TE; TGC & 36 \\
\hline KF; C; DA; ENR; E; CN; LZD; N; QD; RD; TE; TGC & 33 \\
\hline KF; C; CIP; DA; ENR; E; CN; LZD; N; QD; RD; S (HLRA); TE; TGC & $41 ; 45$ \\
\hline
\end{tabular}

High level aminoglycoside resistance (HLAR). 106 isolates that were non-susceptible to gentamicin and/or streptomycin with Kirby-Bauer test, were tested for high level aminoglycoside resistance. None of the tested isolates showed a high level resistance to gentamicin, whereas 19 $(16.5 \%)$ isolates showed high level resistance to streptomycin.

Classification of acquired resistance. Following MDR, XDR and PDR classification, 61 (53\%) strains were classified as MDR and 6 (5.2\%) strains as possibly XDR bacteria (Tab. 2, 3,4 and 5).
Species identification. 67 isolates, characterized as MDR and possibly XDR, were identified by API 20 STREP: 48 strains were identified as E. faecium, 14 as E. faecalis and 5 as E. durans (Tab. 2, 3, 4 and 5).

\section{DISCUSSION}

In this study, expression of antimicrobial resistance was evaluated on strains of Enterococcus spp. isolated from cloacal swabs collected from laying hens raised in small backyard flocks.

As expected, almost all isolates showed resistance to oxacillin, cephalothin, and trimethoprim because of intrinsic resistance to these molecules [6] that were not considered for the evaluation of resistance patterns. Indeed, 101 resistance patterns were identified. This high variability could be related to the high number of tested molecules; however, high variability has been frequently found in other studies performed on poultry enterococci $[12,13]$. Most of the isolates showed a resistance to aminoglycoside molecules, particularly neomycin (100\% of isolates) and streptomycin (93\% of isolates). However, enterococci are intrinsically resistant to clinically achievable concentrations of aminoglycosides due to inability to enter the cell ( $E$. faecalis), and for enzyme-mediated resistance or stericallyhindered ribosome target site (E. faecium). Intrinsic high level resistance to neither gentamicin nor streptomycin has been described in enterococci [6].

HLAR has been tested on all isolates that showed a no-susceptible phenotype in the Kirby-Bauer test for 
Table 3. Resistance patterns of strains identified as E. faecium and classified MDR

\begin{tabular}{|c|c|}
\hline Resistance pattern & Strain \\
\hline DA; ENR; E; N; QD; S; TE & 49 \\
\hline KF; DA; E; N; QD; S; TE & $84 ; 164$ \\
\hline AMP; KF; DA; ENR; N; S; TE & 113 \\
\hline AMP; KF; DA; ENR; E; N; S; TE & 122 \\
\hline KF; CIP; DA; ENR; E; CN; LZD; N; QD; RD; S (HLRA);TE & 101 \\
\hline AMP; KF; CIP; DA; ENR; E; N; QD; RD; S (HLRA); TE & 58 \\
\hline $\mathrm{AMC} ; \mathrm{KF} ; \mathrm{CIP} ; \mathrm{DA} ; \mathrm{ENR} ; \mathrm{E} ; \mathrm{CN} ; \mathrm{N} ; \mathrm{F} ; \mathrm{TE}$ & 80 \\
\hline AMP; KF; DA; ENR; E; CN; N; F; S (HLRA); TE & 110 \\
\hline AMP; KF; CIP; DA; ENR; E; CN; N; QD; S; TE & $108 ; 120$ \\
\hline CIP; DA; ENR; E; CN; N; QD; S; TE & 239 \\
\hline CIP; DA; ENR; E; CN; N; QD; S; TE; TGC & 40 \\
\hline $\mathrm{KF} ; \mathrm{CIP} ; \mathrm{DA} ; \mathrm{ENR} ; \mathrm{E} ; \mathrm{CN} ; \mathrm{N} ; \mathrm{QD} ; \mathrm{S}$; TE & $31 ; 109 ; 168$ \\
\hline AMC; AMP; KF; CIP; DA; ENR; CN; N; QD; S; TGC & 65 \\
\hline$C ; \mathrm{KF} ; \mathrm{DA} ; \mathrm{ENR} ; \mathrm{E} ; \mathrm{N} ; \mathrm{F} ; \mathrm{QD} ; \mathrm{S} ; \mathrm{TE}$ & 57 \\
\hline AMC; KF; C; CIP; DA; ENR; E; CN; LZD; N; F; S & 82 \\
\hline KF; C; CIP; DA; ENR; E; CN; LZD; N; F; QD; S; TE; TGC & 177 \\
\hline AMC; AMP; KF; C; CIP; DA; ENR; E; CN; LZD; N; F; QD; S; TE; TGC & 107 \\
\hline AMC; AMP; KF; CIP; ENR; E; CN; N; F; S; TE & 76 \\
\hline AMC; KF; CIP; DA; ENR; E; CN; N; F; S (HLRA); TE & 79 \\
\hline AMC; AMP; KF; CIP; DA; ENR; E; CN; N; F; S; QD & 86 \\
\hline KF; CIP; DA; ENR; E; N; F; QD; S; TE & 54 \\
\hline $\mathrm{KF} ; \mathrm{CIP} ; \mathrm{DA} ; \mathrm{ENR} ; \mathrm{E} ; \mathrm{N} ; \mathrm{F} ; \mathrm{S} ; \mathrm{TEC}$; TE & 226 \\
\hline KF; CIP; DA; ENR; E; N; F; QD; S; TEC; TE & 162 \\
\hline KF; CIP; DA; ENR; E; CN; LZD; N; S (HLRA) & 190 \\
\hline KF; CIP; DA; ENR; E; LZD; N; QD; S; TE & 118 \\
\hline AMP; KF; CIP; ENR; E; CN; LZD; N; F; S; TE & 104 \\
\hline KF; CIP; ENR; E; CN; LZD; N; F; S; TGC & 91 \\
\hline KF; CIP; DA; ENR; E; CN; LZD; N; F; QD; S & 75 \\
\hline AMC; AMP; KF; CIP; ENR; E; CN; LZD; N; F; S & 78 \\
\hline KF; CIP; DA; ENR; E; CN; LZD; N; F; QD; S (HLRA); TE; TGC & $103 ; 223$ \\
\hline AMP; KF; CIP; DA; ENR; E; CN; LZD; N; F; QD; S; TE; TGC & 114 \\
\hline AMC; AMP; KF; CIP; DA; ENR; E; CN; LZD; N; F; QD; S; TE & 167 \\
\hline KF; DA; ENR; E; CN; LZD; N; F; QD; S; TE; TGC & 175 \\
\hline KF; CIP; DA; ENR; E; LZD; N; F; QD; S; TE; TGC & 158 \\
\hline AMP; KF; CIP; DA; ENR; E; LZD; N; F; QD; S; TE; TGC & 173 \\
\hline AMP; KF; CIP; DA; ENR; E; N; F; RD; S; TE & 94 \\
\hline AMP; KF; C; CIP; DA; ENR; E; CN; LZD; N; F; QD; RD; S; TE & 93 \\
\hline AMP; KF; C; CIP; DA; ENR; E; CN; LZD; N; F; QD; RD; S; TE & 133 \\
\hline KF; C; CIP; DA; ENR; E; CN; LZD; N; F; QD; RD; S (HLRA); TEC; TE & 189 \\
\hline KF; C; CIP; DA; ENR; E; CN; LZD; N; F; QD; RD; S; TEC; TE; TGC & 188 \\
\hline KF; CIP; DA; ENR; E; CN; N; QD; RD; S (HLRA); TEC; TE & 105 \\
\hline KF; CIP; DA; ENR; E; CN; LZD; N; F; QD; RD; S; TEC; TE; TGC & 179 \\
\hline AMC; AMP; KF; CIP; DA; ENR; E; CN; LZD; N; F; QD; RD; S; TE; TGC & 127 \\
\hline
\end{tabular}

streptomycin and/or gentamycin. 19 isolates showed an HLAR to streptomycin, but high level resistance to gentamycin was not observed. In the absence of HLAR, enterococci with lower resistance to cell wall active agents, such as penicillin or ampicillin, may be susceptible to synergistic killing of aminoglycoside-penicillin combination therapy $[6,23]$.
Table 4. Resistance patterns of strains identified as E. durans and classified as MDR

\begin{tabular}{lc}
\hline Resistance pattern & Strain \\
\hline KF; CIP; DA; ENR; E; CN; N; QD; S (HLRA) & $81 ; 119$ \\
\hline AMC; AMP; KF; C; CIP; DA; ENR; E; CN; LZD; N; F; QD; TE; TGC & 157 \\
\hline AMP; CIP; DA; ENR; E; CN; N; F; TE & 160 \\
\hline AMC; AMP; KF; CIP; N; DA; ENR; E; CN; F; QD; RD; TE; TGC & 64 \\
\hline
\end{tabular}

HLAR - High Level Aminoglycoside Resistance

Table 5. Resistance patterns of strains classified as possibly XDR

\begin{tabular}{|c|c|c|}
\hline Resistance pattern & Strain & Species \\
\hline $\begin{array}{l}\text { AMP (HLR); KF; C; DA; ENR; E; LZD; N; QD; S (HLRA); TE; } \\
\text { TGC }\end{array}$ & 87 & E. faecium \\
\hline $\begin{array}{l}\text { KF; C; CIP; DA; ENR; E; CN; LZD; N; F; QD; S (HLRA); TEC; TE; } \\
\text { TGC }\end{array}$ & 42 & E. durans \\
\hline $\begin{array}{l}\text { AMP; KF; C;CIP; DA; ENR; E; CN; LZD; N; QD; RD; S (HLRA); } \\
\text { TEC; TE }\end{array}$ & 98 & E. faecium \\
\hline $\begin{array}{l}\text { AMP; KF; C; CIP; DA; ENR; E; CN; LZD; N; QD; RD; S (HLRA); } \\
\text { TEC; TE; TGC }\end{array}$ & 90 & E. faecium \\
\hline $\begin{array}{l}\text { AMC; AMP; KF; C; CIP; DA; ENR; E; CN; LZD; N; F; QD; RD; } \\
\text { TEC; TE; TGC }\end{array}$ & 128 & E. faecium \\
\hline $\begin{array}{l}\text { AMC; AMP; KF; C; CIP; DA; ENR; E; CN; LZD; N; F; QD; RD; } \\
\text { TEC;TE; TGC }\end{array}$ & 222 & E. durans \\
\hline
\end{tabular}

HLAR - High Level Aminoglycoside Resistance

Regarding erythromycin, $87.8 \%$ of isolates was nosusceptible, similar to other studies on enterococci isolated from turkeys [18] and broiler chickens [8, 11, 12, 13, 16, 17]. Also in the report of European Food Safety Authorities regarding years 2005-2011, macrolide resistance was the most frequently observed in poultry enterococci [19]. Cross-resistance among macrolide-lincosamide-streptogramin $\left(M L S_{B}\right.$ resistance) is well characterized in enterococci and 3 mechanisms of acquired resistance have been described: methylation of $23 \mathrm{~S}$ rRNA, active efflux or inactivating enzymes $[6,18]$. In the current study, 45 isolates showed a concomitant resistance to erythromycin, clindamycin and quinupristin-dalfopristin, suggesting an acquired MLS $_{\mathrm{B}}$ resistance.

Diffuse resistance was detected to fluoroquinolones: $86.1 \%$ of isolates were non-susceptible to enrofloxacin and $70.4 \%$ were non-susceptible to ciprofloxacin. Fluoroquinoles have been introduced on a large scale in veterinary therapy in recent years, leading to a high prevalence of resistant enterococcal isolates in certain animal groups [8].

Resistance to tetracycline has been detected in $65.2 \%$ of isolated strains. Tetracycline resistance has an importance due to its association with other antimicrobial molecules resistances [4].

In agreement with other authors [16], moderate resistance was observed to rifampicin and chloramphenicol, but these molecules are poorly used in the treatment of Enterococcus infections [6].

Moderate resistance was observed for linezolid and tigecycline. The mechanisms of tigecycline resistance are unknown, whereas linezolid resistance can be mediated by mutation or an acquired gene that can be transferred from staphylococci to enterococci [6].

Glycopeptide resistance, mainly to vancomycin, has raised much research interest during last 25 years, because vancomycin is considered a drug of 'last resort' in human medicine for the treatment of infections by multi-resistant 
enterococci and methicillin resistant Staphylococcus aureus [5]. In the presented study, a low percentage of isolates showed resistance to teicoplanin and vancomycin with the KirbyBauer test. MIC did not confirm the resistance to vancomycin, suggesting that the results of the Kirby-Bauer method for this antibiotic must be confirmed by other tests [25].

61 of the isolates in the current were classified as MDR, and 6 as possibly XDR bacteria. The strains have been identified as E. faecium, E. faecalis and E. durans, all potential pathogens for humans [1]. E. faecium strains are usually hostspecific, but this species could act as a very efficient donor of antimicrobial resistance genes to other enterococci, or more pathogenic bacteria, as well as between different hosts. The same clonal multidrug resistant $E$. faecalis strains have been detected in animals and humans affected by sepsis and endocarditis, suggesting a direct risk of infection from animal to humans [26].

\section{CONCLUSIONS}

The presence of multidrug resistant enterococci in hobby poultry flocks could represent a hazard for public health, considering the close contact between humans and animals. Contamination of the environment and human furniture with the faeces of poultry harbouring resistant enterococci is a risk for all members of the farm family, including children and elders, providing a conduit for the entrance of resistance genes into the community where further transmission is possible.

Backyard poultry flocks have more risk factors compared to commercial ones. Hens and broilers, kept for personal consumption of eggs and meat, usually share the same outside environment with their owners. Moreover, eggs from hobby poultry are not submitted to dipping, thus faeces contamination of shells may facilitate enterococci infection of consumers.

Backyard poultry could be a source of infection also for other domestic animals, such as dogs, cat or pigeons, that can further infect humans or amplify the distribution of resistant strains in the environment.

In conclusion, the presented study, although performed in a restricted geographical area and on a limited number of birds, contribute to the verification of the presence of multi-drug resistant enterococci in the environment, and determination of their antimicrobial resistance profiles. The obtained results underline that not only commercially-kept poultry, but also backyard birds could have an epidemiological role in the amplification and transmission of antimicrobial resistant bacteria.

\section{REFERENCES}

1. Byappanahalli MN, Nevers MB, Korajkic A, Staley ZR, Harwood VJ. Enterococci in the environment. Microbiol Mol Biol Rev. 2012; 4: 685-706.

2. Domig KJ, Mayer HK, Kneifel W. Methods used for the isolation, enumeration, characterization and identification of Enterococcus spp. 1. Media for isolation and enumeration. Int J Food Microbiol. 2003; 88: 147-164.

3. Hegstad K, Mikalsen T, Coque TM, Werner G, Sundsfjord A. Mobile genetics elements and their contribution to the emergence of antimicrobial resistant Enterococcus faecalis and Enterococcus faecium. Clin Microbiol Infect. 2010; 16: 541-554.

4. Hammerum AM. Enterococci of animal origin and their significance for public health. Clin Microbiol Infect. 2012; 18: 619-625.
5. Nilsson O. Vancomycin resistant enterococci in farm animalsoccurrence and importance. Infect Ecol Epidemiol. 2012; 2: doi10.3402/ iee.v2i0.16959..

6. Hollenbeck BL, Rice LB. Intrinsic and acquired resistance mechanisms in enterococcus. Virulence 2012; 5: 421-433.

7. Robbins KM, Suyemoto MM, Lyman RL, Martin MP, Barnes HJ, Borst LB. An outbreak and source investigation of enterococcal spondylitis in broiler caused by Enterococcus cecorum. Avian Dis. 2012; 56: 768-773.

8. Šeputienè V, Bogdaitė A, Ružauskas M, Sužiedèlienè E. Antibiotic resistance genes and virulence factors in Enterococcus faecium and Enterococcus faecalis from diseased farm animals: pigs, cattle and poultry. Pol J Vet Sci. 2012; 3: 431-438.

9. Sørensen TL, Blom M, Monnet DL, Frimodt-Møller N, Poulsen RL, Espersen F. Transient intestinal carriage after ingestion of antibioticresistant Enterococcus faecium from chicken and pork. N Engl J Med. 2001; 16: 1161-1166.

10. Marshall BM, Levy SB. Food animals and antimicrobials: impacts on human health. Clin Microbiol Rev. 2011; 4: 718-733.

11. Obeng AS, Rickard H, Ndi O, Sexton M, Barton M. Comparison of antimicrobial resistance patterns in enterococci from intensive and free range chickens in Australia. Avian Pathol. 2013; 1: 45-54.

12. Diarra MS, Rempel H, Champagne J, Masson L, Pritchard J, Topp E. Distribution of antimicrobial resistance and virulence genes in Enterococcus spp. and characterization of isolates from broiler chickens. Appl Environ Microbiol. 2010; 24: 8033-8043.

13. Furtula V, Jackson CR, Farrell EG, Barrett JB, Hiott LM, Chambers PA. Antimicrobial resistance in Enterococcus spp. isolated from environmental samples in an area of intensive poultry production. Int J Environ Public Health. 2013; 10: 1020-1036.

14. Getachew Y, Hassan L, Zakaria Z, Abdul Aziz S. Genetic variability of vancomycin-resistant Enterococcus faecium and Enterococcus faecalis isolates from humans, chickens, and pigs in Malaysia. Appl Environ Microbiol. 2013; 79: 4528-4533.

15. Nilsson O, Greko C, Bengtsson B. Environmental contamination by vancomycin resistant enterococci (VRE) in Swedish broiler production. Acta Vet Scand. 2009; 51: 49.

16. Aarestrup FM, Agerso Y, Gerner-Smidt P, Madsen M, Jensen LB. Comparison of antimicrobial resistance phenotypes and resistance genes in Enterococcus faecalis and Enterococcus faecium from humans in the community, broilers, and pigs in Denmark. Diagn Microbiol Infect Dis. 2000; 37: 127-137.

17. Petsaris O, Miszczak F, Gicquel-Bruneau M, Perrin-Guyomard A, Humbert F, Sanders P, Leclercq R. Combined antimicrobial resistance in Enterococcus faecium isolated from chickens. Appl Environ Microbiol. 2005; 5: 2796-2799.

18. Jaglic Z, Vlkova H, Bardon J, Michu E, Cervinkova D, Babak V. Distribution, characterization and genetic bases of erythromycin resistance in staphylococci and enterococci originating from livestock. Zoonoses Pub Health. 2012; 59: 202-211.

19. Garcia-Migura L, Hendriksen RS, Fraile L, Aarestrup FM. Antimicrobial resistance of zoonotic and commensal bacteria in Europe: the missing link between consumption and resistance in veterinary medicine. Vet Microbiol. 2014; 170: 1-9.

20. Bauer A, Kirby WMN, Sherris JC, Turk M. Antibiotic susceptibility testing by a standardized single disc method. Am J Pathol. 1966; 45: 493-496.

21. Clinical and Laboratory Standards Institute. Performance standards for antimicrobial susceptibility testing, Seventeenth Informational Supplement. Clinical and Laboratory Standards Institute document M100-S17 [ISBN 1-56238-625-5]. Clinical and Laboratory Standards Institute, 940 West Valley Road, Suite 1400, Wayne, Pennsylvania 19087-1898 USA, 2007.

22. EUCAST. The European Committee on Antimicrobial Susceptibility Testing. Breakpoint tables for interpretation of MICs and zone diameters. Version 3.1, 2013. http://www.eucast.org

23. Clinical and Laboratory Standards Institute. Methods for Dilution Antimicrobial Susceptibility Tests for Bacteria That Grow Aerobically; Approved Standard-Seventh Edition. Clinical and Laboratory Standards Institute document M7-A7 [ISBN 1-56238-587-9]. Clinical and Laboratory Standards Institute, 940 West Valley Road, Suite 1400, Wayne, Pennsylvania 19087-1898 USA, 2006.

24. Magiorakos AP, Srinivasan A, Carey RB, Carmeli Y, Falagas ME, Giske CG, et al. Multidrug-resistant, extensively drug-resistant and pandrug-resistant bacteria: an international expert proposal for interim standard definitions for acquired resistance. Clin Microbiol Infect. 2012; 18: 268-281.

25. Andrews JM. Determination of minimum inhibitory concentrations. J Antimicrob Chemother. 2001; 48 Suppl 1: 5-16.

26. Hammerum AM, Lester CH, Heuer OE. Antimicrobial-resistant enterococci in animals and meat: a human health hazard? Foodborne Pathog Dis. 2010; 10: 1137-1146. 